\title{
Homogeneous Lorentzian Structures on the Oscillator groups *
}

\author{
P.M. GADEA AND J.A. OUBIÑA ${ }^{\dagger}$
}

\begin{abstract}
We obtain all the homogeneous pseudo-Riemannian structures on the oscillator groups equipped with a family of left-invariant Lorentzian metrics. Moreover, in the 4-dimensional case we determine all the corresponding reductive decompositions and groups of isometries.
\end{abstract}

\section{Introduction}

In [7] Medina proved that the oscillator groups are, except for direct extensions with Euclidean groups, the only non-commutative simply connected solvable Lie groups which admit a bi-invariant Lorentzian metric. From the biinvariance of the metric, it turns out that the corresponding pseudo-Riemannian spaces are symmetric. These groups also appear in various types of problems which arise from mathematical physics. For instance, the Lie algebra of the 4-dimensional oscillator group is associated to the harmonic oscillator problem (see Streater [11], where the group is so named because of this property) and, on the other hand, this Lorentzian symmetric space-time has been found to be a special case of solutions of the Einstein-Yang-Mills equations (see Levichev [5]).

It is a well-known fact that under certain topological conditions, a pseudoRiemannian symmetric space is characterized by the vanishing of the covariant derivative of the curvature. In the homogeneous Riemannian case, Ambrose and Singer [1] extended that characterization. They proved that a connected, simply connected and complete Riemannian manifold $(M, g)$ is homogeneous if and only if there exists a $(1,2)$ tensor field $S$ on $M$ (called a homogeneous Riemannian structure) satisfying certain properties (see (2.1)). In [2] we have extended the Ambrose-Singer characterization to the case of pseudo-Riemannian manifolds, introducing homogeneous pseudo-Riemannian structures. We proved that a connected, simply connected and geodesically complete pseudo-Riemannian manifold $(M, g)$ admits a homogeneous pseudo-Riemannian structure if and only if it is a reductive homogeneous pseudo-Riemannian space. Furthermore, in [3]

\footnotetext{
*MS Classification (1991): 53C30, 53C50, 22E35. Key Words: Homogeneous structures, pseudo-Riemannian manifolds, oscillator groups.

${ }^{\dagger}$ Partially supported by DGES (Spain) under Project PB95-0124 and by Xunta de Galicia under Project XUGA 20703B98.
} 
we have obtained a classification of homogeneous pseudo-Riemannian structures into eight classes similar to the Tricerri-Vanhecke classification [12] for the Riemannian case. If the signature of the metric is $(k, n-k)$, those classes are defined by the subspaces of certain space $\mathcal{S}_{1} \oplus \mathcal{S}_{2} \oplus \mathcal{S}_{3}$ which are invariant under the action of the pseudo-orthogonal group $O_{k}(n)$. The trivial class corresponds to the symmetric pseudo-Riemannian spaces.

In this paper, we consider a family of Lorentzian left-invariant metrics on the oscillator groups which generalize those ones introduced by Levichev [6] in his study of causal homogeneous Lorentzian 4-manifolds. All the corresponding pseudo-Riemannian spaces except one are not symmetric, and our purpose is to study their homogeneity by means of their homogeneous pseudo-Riemannian structures. The contents of this paper are as follows. In $\S 2$ we recall some results about homogeneous pseudo-Riemannian structures. In $\S 3$ we give the formulas for the Levi-Civita connections and curvatures of a family of left-invariant Lorentzian metrics on the $(2 m+2)$-dimensional oscillator group $G\left(\lambda_{1}, \ldots, \lambda_{m}\right)$, $\lambda_{1}, \ldots, \lambda_{m} \in \mathbb{R}^{+}$. In $\S 4$ we obtain the general expressions for the homogeneous pseudo-Riemannian structures on these Lorentzian manifolds. Finally, in $\S 5$ we determine all the reductive decompositions associated to each homogeneous Lorentzian structure in the nonsymmetric 4-dimensional cases and we obtain all the corresponding groups of isometries.

\section{Homogeneous pseudo-Riemannian structures}

Let $(M, g)$ be a connected $C^{\infty}$ pseudo-Riemannian manifold of dimension $n$ and signature $(k, n-k)$. Let $\nabla$ be the Levi-Civita connection of $g$ and $R$ the curvature tensor field, for which we adopt the conventions $R_{X Y} Z=\nabla_{[X, Y]} Z-$ $\nabla_{X} \nabla_{Y} Z+\nabla_{Y} \nabla_{X} Z, R_{X Y Z W}=g\left(R_{X Y} Z, W\right)$, for $X, Y, Z, W \in \mathfrak{X}(M)$.

A homogeneous pseudo-Riemannian structure on $(M, g)$ is [2] a tensor field $S$ of type $(1,2)$ on $M$ such that the connection $\widetilde{\nabla}=\nabla-S$ satisfies

$$
\widetilde{\nabla} g=0, \quad \widetilde{\nabla} R=0, \quad \widetilde{\nabla} S=0 .
$$

If $g$ is a Lorentzian metric $(k=1)$, we say that $S$ is a homogeneous Lorentzian structure. In [2] we have proved that if $(M, g)$ is connected, simply connected and geodesically complete then it admits a homogeneous pseudo-Riemannian structure if and only if it is a reductive homogeneous pseudo-Riemannian manifold.

Let $V$ be a real vector space endowed with an inner product $\langle$,$\rangle of signature$ $(k, n-k)$. The space $(V,\langle\rangle$,$) is the model for each tangent space T_{x} M, x \in M$, of a reductive homogeneous pseudo-Riemannian manifold of signature $(k, n-k)$. Consider the vector space $\mathcal{S}(V)$ of tensors of type $(0,3)$ on $(V,\langle\rangle$,$) satisfying$ the same symmetries as those of a homogeneous pseudo-Riemannian structure $S$, that is, $\mathcal{S}(V)=\left\{S \in \otimes^{3} V^{*}: S_{X Y Z}=-S_{X Z Y}, X, Y, Z \in V\right\}$, where $S_{X Y Z}=\left\langle S_{X} Y, Z\right\rangle$. The inner product of $V$ induces in a natural way an inner product in $\mathcal{S}(V)$, given by $\left\langle S, S^{\prime}\right\rangle=\sum_{i, j, k=1}^{n} \varepsilon_{i} \varepsilon_{j} \varepsilon_{k} S_{e_{i} e_{j} e_{k}} S_{e_{i} e_{j} e_{k}}^{\prime}$, where $\left\{e_{i}\right\}$ is 
an orthonormal basis of $V,\left\langle e_{i}, e_{i}\right\rangle=\varepsilon_{i}, \varepsilon_{i}=-1$ if $1 \leq i \leq k, \varepsilon_{i}=1$ if $k+1 \leq$ $i \leq n$. In [3], we have established the decomposition of $\mathcal{S}(V)$ into invariant and irreducible subspaces under the action of the pseudo-orthogonal group $O_{k}(n)$ given by $(a S)_{X Y Z}=S_{a^{-1} X a^{-1} Y a^{-1} Z}, a \in O_{k}(n)$. If $c_{12}: \mathcal{S}(V) \rightarrow V^{*}$ is the map defined by

$$
c_{12}(S)(Z)=\sum_{i=1}^{n} \varepsilon_{i} S_{e_{i} e_{i} Z}, \quad Z \in V
$$

where $\left\{e_{i}\right\}$ is an orthonormal basis of $V$ as above, we have

Theorem 2.1. If $\operatorname{dim} V \geq 3$, then $\mathcal{S}(V)$ decomposes into the orthogonal direct sum of subspaces which are invariant and irreducible under the action of $O_{k}(n)$, $\mathcal{S}(V)=\mathcal{S}_{1}(V) \oplus \mathcal{S}_{2}(V) \oplus \mathcal{S}_{3}(V)$, where

$$
\begin{aligned}
& \mathcal{S}_{1}(V)=\left\{S \in \mathcal{S}(V): S_{X Y Z}=\langle X, Y\rangle \omega(Z)-\langle X, Z\rangle \omega(Y), \omega \in V^{*}\right\}, \\
& \mathcal{S}_{2}(V)=\left\{S \in \mathcal{S}(V): \mathcal{S}_{X Y Z} S_{X Y Z}=0, c_{12}(S)=0\right\}, \\
& \mathcal{S}_{3}(V)=\left\{S \in \mathcal{S}(V): S_{X Y Z}+S_{Y X Z}=0\right\} . \\
& \mathcal{S}_{1}(V) \oplus \mathcal{S}_{2}(V)=\left\{S \in \mathcal{S}(V): \mathcal{S}_{X Y Z} S_{X Y Z}=0\right\} \\
& \mathcal{S}_{1}(V) \oplus \mathcal{S}_{3}(V)=\left\{S \in \mathcal{S}(V): S_{X Y Z}+S_{Y X Z}=2\langle X, Y\rangle \omega(Z)\right. \\
&\left.\quad-\langle X, Z\rangle \omega(Y)-\langle Y, Z\rangle \omega(X), \omega \in V^{*}\right\}, \\
& \mathcal{S}_{2}(V) \oplus \mathcal{S}_{3}(V)=\left\{S \in \mathcal{S}(V): c_{12}(S)=0\right\} .
\end{aligned}
$$

Moreover, $\operatorname{dim} \mathcal{S}_{1}(V)=n, \operatorname{dim} \mathcal{S}_{2}(V)=n\left(n^{2}-4\right) / 3, \operatorname{dim} \mathcal{S}_{3}(V)=n(n-1)(n-$ 2)/6 and $\operatorname{dim} \mathcal{S}(V)=n^{2}(n-1) / 2$. If $\operatorname{dim} V=2$ then $\mathcal{S}(V)=\mathcal{S}_{1}(V)$.

We say that the homogeneous pseudo-Riemannian structure $S$ on $(M, g)$ is of type $\{0\}, \mathcal{S}_{i}(i=1,2,3)$ or $\mathcal{S}_{i} \oplus \mathcal{S}_{j}(1 \leq i<j \leq 3)$ if, for each point $x \in M$, $S(x) \in \mathcal{S}\left(T_{x} M\right)$ belongs to $\{0\}, \mathcal{S}_{i}\left(T_{x} M\right)$ or $\left(\mathcal{S}_{i} \oplus \mathcal{S}_{j}\right)\left(T_{x} M\right)$, respectively.

\section{The oscillator groups}

Let $\lambda_{1}, \ldots, \lambda_{m}$ be $m$ positive real numbers and $\lambda=\left(\lambda_{1}, \ldots, \lambda_{m}\right)$. The oscillator algebra $\mathfrak{g}_{m}(\lambda)=\mathfrak{g}\left(\lambda_{1}, \ldots, \lambda_{m}\right)$ is defined as the real Lie algebra with $2 m+2$ generators $P, X_{1}, \ldots, X_{m}, Y_{1}, \ldots, Y_{m}, Q$, with nonzero brackets (see $[7,8,10]$ )

$$
\left[X_{j}, Y_{j}\right]=P, \quad\left[Q, X_{j}\right]=\lambda_{j} Y_{j}, \quad\left[Q, Y_{j}\right]=-\lambda_{j} X_{j}, \quad 1 \leq j \leq m .
$$

That is, $\mathfrak{g}_{m}(\lambda)$ is the semidirect product of the Heisenberg algebra $\mathfrak{h}_{m}$ generated by $P, X_{1}, \ldots, X_{m}, Y_{1}, \ldots, Y_{m}$, and the line generated by $Q$, under the homomorphism $\operatorname{ad}_{\mid \mathfrak{h}_{m}}:\langle Q\rangle \rightarrow \operatorname{Der}\left(\mathfrak{h}_{m}\right)$. It is a solvable non-nilpotent Lie algebra and the connected simply connected Lie group whose Lie algebra is $\mathfrak{g}_{m}(\lambda)$ is the oscillator group $G_{m}(\lambda)=G\left(\lambda_{1}, \ldots, \lambda_{m}\right)$.

If we identify the $(2 m+1)$-dimensional Heisenberg group $H_{m}$ with the manifold $\mathbb{R} \times \mathbb{C}^{m}$ equipped with the product

$$
\left(p, z_{1}, \ldots, z_{m}\right)\left(p^{\prime}, z_{1}^{\prime}, \ldots, z_{m}^{\prime}\right)=\left(p+p^{\prime}+\frac{1}{2} \sum_{j=1}^{m} \operatorname{Im}\left(\bar{z}_{j} z_{j}^{\prime}\right), z_{1}+z_{1}^{\prime}, \ldots, z_{m}+z_{m}^{\prime}\right)
$$


then the oscillator group $G_{m}(\lambda)$ may be described as the semidirect product $H_{m} \times_{\alpha} \mathbb{R}$, where the action $\alpha: H_{m} \times \mathbb{R} \rightarrow H_{m}$ is given by $\alpha_{q}\left(p, z_{1}, \ldots, z_{m}\right)=$ $\left(p, e^{i \lambda_{1} q} z_{1}, \ldots, e^{i \lambda_{m} q} z_{m}\right), q \in \mathbb{R}$. Thus, the group operation in $G_{m}(\lambda)$ is

$$
\begin{aligned}
& \left(p, z_{1}, \ldots, z_{m}, q\right)\left(p^{\prime}, z_{1}^{\prime}, \ldots, z_{m}^{\prime}, q^{\prime}\right)= \\
& \quad\left(p+p^{\prime}+\frac{1}{2} \sum_{j=1}^{m} \operatorname{Im}\left(\bar{z}_{j} e^{i \lambda_{j} q} z_{j}^{\prime}\right), z_{1}+e^{i \lambda_{1} q} z_{1}^{\prime}, \ldots, z_{m}+e^{i \lambda_{m} q} z_{m}^{\prime}, q+q^{\prime}\right) .
\end{aligned}
$$

We consider on $G_{m}(\lambda)$ the family of left-invariant Lorentzian metrics $g_{\varepsilon}$, $-1<\varepsilon<1$, with nonvanishing inner products $\langle,\rangle_{\varepsilon}$ on $\mathfrak{g}_{m}(\lambda)$ given by

$$
\langle P, P\rangle_{\varepsilon}=\langle Q, Q\rangle_{\varepsilon}=\varepsilon, \quad\langle P, Q\rangle_{\varepsilon}=1, \quad\left\langle X_{i}, X_{j}\right\rangle_{\varepsilon}=\left\langle Y_{i}, Y_{j}\right\rangle_{\varepsilon}=\delta_{i j}
$$

If $\varepsilon=0$ and $\lambda_{i}=1$ for each $i=1, \ldots, m$, the corresponding Lorentzian metric is also right-invariant. In the other cases, $g_{\varepsilon}$ is not bi-invariant.

The Levi-Civita connection is given by $2\left\langle\nabla_{U} V, W\right\rangle_{\varepsilon}=\langle[U, V], W\rangle_{\varepsilon}-\langle[V, W]$, $U\rangle_{\varepsilon}+\langle[W, U], V\rangle_{\varepsilon}$ for all $U, V, W \in \mathfrak{g}_{m}(\lambda)$. So, we obtain that the not always null covariant derivatives between generators are

$$
\begin{array}{lll}
\nabla_{P} X_{j}=-\frac{\varepsilon}{2} Y_{j}=\nabla_{X_{j}} P, & \nabla_{X_{j}} Q=-\frac{1}{2} Y_{j}, & \nabla_{Q} X_{j}=\left(\lambda_{j}-\frac{1}{2}\right) Y_{j}, \\
\nabla_{P} Y_{j}=\frac{\varepsilon}{2} X_{j}=\nabla_{Y_{j}} P, & \nabla_{Y_{j}} Q=\frac{1}{2} X_{j}, & \nabla_{Q} Y_{j}=-\left(\lambda_{j}-\frac{1}{2}\right) X_{j}, \\
\nabla_{X_{j}} Y_{k}=\frac{1}{2} \delta_{j k} P=-\nabla_{Y_{k}} X_{j}, &
\end{array}
$$

and the not always null components of the curvature tensor field are given by

$$
\begin{array}{ll}
R_{P X_{j}} P=\frac{\varepsilon^{2}}{4} X_{j}, & R_{P Y_{j}} P=\frac{\varepsilon^{2}}{4} Y_{j}, \\
R_{P X_{j}} X_{k}=-\frac{\varepsilon}{4} \delta_{j k} P, & R_{P Y_{j}} Y_{k}=-\frac{\varepsilon}{4} \delta_{j k} P, \\
R_{P X_{j}} Q=\frac{\varepsilon}{4} X_{j}, & R_{P Y_{j}} Q=\frac{\varepsilon}{4} Y_{j}, \\
R_{X_{j} Q} P=-\frac{\varepsilon}{4} X_{j}, & R_{Y_{j} Q} P=-\frac{\varepsilon}{4} Y_{j}, \\
R_{X_{j} Q} X_{k}=\frac{1}{4} \delta_{j k} P, & R_{Y_{j} Q} Y_{k}=\frac{1}{4} \delta_{j k} P, \\
R_{X_{j} Q} Q=-\frac{1}{4} X_{j}, & R_{Y_{j} Q} Q=-\frac{1}{4} Y_{j}, \\
R_{X_{i} X_{j}} Y_{k}=\frac{\varepsilon}{4}\left(\delta_{j k} Y_{i}-\delta_{i k} Y_{j}\right), & R_{Y_{i} Y_{j}} X_{k}=\frac{\varepsilon}{4}\left(\delta_{j k} X_{i}-\delta_{i k} X_{j}\right), \\
R_{X_{j} Y_{k}} X_{i}=-\frac{\varepsilon}{2}\left(\delta_{j k} Y_{i}+\frac{1}{2} \delta_{i k} Y_{j}\right), & \\
R_{X_{j} Y_{k}} Y_{i}=\frac{\varepsilon}{2}\left(\delta_{j k} X_{i}+\frac{1}{2} \delta_{i j} X_{k}\right) . &
\end{array}
$$

\section{Homogeneous Lorentzian structures on $G_{m}(\lambda)$}

We shall determine the homogeneous Lorentzian structures on $G_{m}(\lambda)$ in terms of the basis $\left\{\eta, \alpha^{1}, \ldots, \alpha^{m}, \beta^{1} \ldots, \beta^{m}, \xi\right\}$ dual to $\left\{P, X_{1}, \ldots, X_{m}, Y_{1}, \ldots, Y_{m}, Q\right\}$. If $S$ is a homogeneous Lorentzian structure on $\left(G_{m}(\lambda), g_{\varepsilon}\right)$ and $\widetilde{\nabla}=\nabla-S$, then the condition $\widetilde{\nabla} g=0$ in (2.1) is equivalent to $S_{W U V}+S_{W V U}=0$ for all 
$W, U, V \in \mathfrak{g}_{m}(\lambda)$. Moreover, $\widetilde{\nabla} R=0$ is equivalent to the condition

$$
\begin{aligned}
\left(\nabla_{W} R\right)\left(V_{1}, V_{2}, V_{3}, V_{4}\right)= & -R\left(S_{W} V_{1}, V_{2}, V_{3}, V_{4}\right)-R\left(V_{1}, S_{W} V_{2}, V_{3}, V_{4}\right) \\
& -R\left(V_{1}, V_{2}, S_{W} V_{3}, V_{4}\right)-R\left(V_{1}, V_{2}, V_{3}, S_{W} V_{4}\right),
\end{aligned}
$$

for all $W, V_{1}, V_{2}, V_{3}, V_{4} \in \mathfrak{g}_{m}(\lambda)$. Substituting $\left(V_{1}, V_{2}, V_{3}, V_{4}\right)$ by $\left(P, X_{j}, P, Q\right)$, $\left(X_{j}, Y_{j}, Y_{j}, Q\right),\left(P, Y_{j}, P, Q\right)$ and $\left(X_{j}, Y_{j}, X_{j}, Q\right)$, we obtain, respectively,

$$
\begin{array}{ll}
\varepsilon S_{W P X_{j}}+\varepsilon^{2} S_{W X_{j} Q}=0, & S_{W P X_{j}}-3 \varepsilon S_{W X_{j} Q}=2 \varepsilon \beta^{j}(W), \\
\varepsilon S_{W P Y_{j}}+\varepsilon^{2} S_{W Y_{j} Q}=0, & S_{W P Y_{j}}-3 \varepsilon S_{W Y_{j} Q}=-2 \varepsilon \alpha^{j}(W) .
\end{array}
$$

From these equations we have

$$
\begin{gathered}
S_{W P X_{j}}=\frac{\varepsilon}{2} \beta^{j}(W), \quad S_{W P Y_{j}}=-\frac{\varepsilon}{2} \alpha^{j}(W), \\
\varepsilon\left(S_{W X_{j} Q}+\frac{1}{2} \beta^{j}(W)\right)=0, \quad \varepsilon\left(S_{W Y_{j} Q}-\frac{1}{2} \alpha^{j}(W)\right)=0 .
\end{gathered}
$$

Replacing $\left(V_{1}, V_{2}, V_{3}, V_{4}\right)$ in (4.1) by $\left(X_{j}, X_{k}, X_{j}, Y_{j}\right)$ and $\left(X_{i}, X_{j}, Y_{i}, Y_{k}\right)$, with $j \neq k$, we obtain, respectively,

$$
\varepsilon\left(S_{W X_{j} Y_{k}}-S_{W X_{k} Y_{j}}\right)=0, \quad \varepsilon\left(S_{W X_{j} X_{k}}-S_{W Y_{j} Y_{k}}\right)=0 .
$$

Finally, replacing $\left(V_{1}, V_{2}, V_{3}, V_{4}\right)$ in (4.1) by $\left(X_{j}, Q, X_{j}, Q\right)$, we obtain

$$
S_{W P Q}=0 .
$$

It is easy to see that the condition $\widetilde{\nabla} R=0$ in $(2.1)$ is satisfied if and only if the equations (4.2), (4.3), (4.4) and (4.5) are satisfied for all $W \in \mathfrak{g}_{m}(\lambda)$. We put

$$
\begin{gathered}
\theta_{j k}(W)=S_{W X_{j} Y_{k}}, \quad \mu_{j k}(W)=S_{W X_{j} X_{k}}, \quad \nu_{j k}(W)=S_{W Y_{j} Y_{k}}, \\
\rho_{j}(W)=S_{W X_{j} Q}, \quad \sigma_{j}(W)=S_{W Y_{j} Q},
\end{gathered}
$$

for $1 \leq j, k \leq m$. We have $\mu_{j k}=-\mu_{k j}$ and $\nu_{j k}=-\nu_{k j}$. Now, we shall determine the conditions for the 1-forms $\theta_{j k}, \mu_{j k}, \nu_{j k}, \rho_{j}$ and $\sigma_{j}$ under which the condition $\widetilde{\nabla} S=0$ in (2.1) is satisfied.

By (4.2), (4.5), (4.6) and (4.7), the connection $\widetilde{\nabla}=\nabla-S$ is given by

$$
\begin{gathered}
\widetilde{\nabla}_{Z} P=0, \quad \widetilde{\nabla}_{Z} Q=\sum_{i}\left(\rho_{i}+\frac{1}{2} \beta^{i}\right)(Z) X_{i}+\sum_{i}\left(\sigma_{i}-\frac{1}{2} \alpha^{i}\right)(Z) Y_{i}, \\
\widetilde{\nabla}_{Z} X_{j}=-\left(\rho_{j}+\frac{1}{2} \beta^{j}\right)(Z) P+\sum_{i} \mu_{i j}(Z) X_{i}-\sum_{i} \theta_{j i}(Z) Y_{i}+\left(\left(\lambda_{j}-\frac{1}{2}\right) \xi-\frac{\varepsilon}{2} \eta\right)(Z) Y_{j}, \\
\widetilde{\nabla}_{Z} Y_{j}=-\left(\sigma_{j}-\frac{1}{2} \alpha^{j}\right)(Z) P+\sum_{i} \theta_{i j}(Z) X_{i}+\left(\frac{\varepsilon}{2} \eta-\left(\lambda_{j}-\frac{1}{2}\right) \xi\right)(Z) X_{j}+\sum_{i} \nu_{i j}(Z) Y_{i},
\end{gathered}
$$

for every $Z \in \mathfrak{g}_{m}(\lambda)$. Then, replacing $\left(V_{1}, V_{2}\right)$ in the equation $\left(\widetilde{\nabla}_{Z} S\right)\left(W, V_{1}, V_{2}\right)$ $=0$ by $\left(X_{j}, Y_{k}\right),\left(X_{j}, X_{k}\right),\left(Y_{j}, Y_{k}\right),\left(X_{j}, Q\right)$ and $\left(Y_{j}, Q\right)$ we obtain, respectively, 
being $\Lambda_{j}=\lambda_{j}-\frac{1}{2}$,

$$
\begin{array}{r}
\widetilde{\nabla} \theta_{j k}=\sum_{i}\left(\theta_{i k} \wedge \mu_{j i}+\nu_{i k} \wedge \theta_{j i}\right)+\Lambda_{j} \xi \otimes \nu_{j k}-\Lambda_{k} \xi \otimes \mu_{j k} \\
\widetilde{\nabla} \mu_{j k}=\sum_{i}\left(\mu_{i k} \wedge \mu_{j i}+\theta_{j i} \wedge \theta_{k i}\right)+\Lambda_{k} \xi \otimes \theta_{j k}-\Lambda_{j} \xi \otimes \theta_{k j} \\
\widetilde{\nabla} \nu_{j k}=\sum_{i}\left(\nu_{i k} \wedge \nu_{j i}+\theta_{i j} \wedge \theta_{i k}\right)+\Lambda_{k} \xi \otimes \theta_{k j}-\Lambda_{j} \xi \otimes \theta_{j k} \\
\widetilde{\nabla} \rho_{j}=\sum_{i}\left(\rho_{i} \wedge \mu_{j i}+\sigma_{i} \wedge \theta_{j i}\right)+\frac{1}{2} \sum_{i}\left(\beta^{i} \otimes \mu_{j i}-\alpha^{i} \otimes \theta_{j i}\right)+\left(\Lambda_{j} \xi-\frac{\varepsilon}{2} \eta\right) \otimes \sigma_{j}, \\
\widetilde{\nabla} \sigma_{j}=\sum_{i}\left(\sigma_{i} \wedge \nu_{j i}-\rho_{i} \wedge \theta_{i j}\right)+\frac{1}{2} \sum_{i}\left(\alpha^{i} \otimes \nu_{i j}-\beta^{i} \otimes \theta_{i j}\right)-\left(\Lambda_{j} \xi-\frac{\varepsilon}{2} \eta\right) \otimes \rho_{j} .
\end{array}
$$

In the case of the bi-invariant metric $\left(\varepsilon=0\right.$ and $\lambda_{i}=1$ for each $\left.i=1, \ldots, m\right)$, the oscillator group is a Lorentzian symmetric space and the tensor field $S=0$ is a homogeneous Lorentzian structure on $\left(G_{m}(\lambda), g_{0}\right)$. Moreover, from (4.2), (4.5), (4.6) and (4.7), we deduce

Theorem 4.1. All the homogeneous Lorentzian structures on the oscillator group $G_{m}(\lambda)$ with the left-invariant Lorentzian metric $g_{0}$ are given by

$$
\begin{aligned}
S=\sum_{i=1}^{m}\left(\rho_{i} \otimes\left(\alpha^{i} \wedge \xi\right)+\sigma_{i} \otimes\left(\beta^{i} \wedge \xi\right)\right)+\sum_{j, k=1}^{m} \theta_{j k} \otimes\left(\alpha^{j} \wedge \beta^{k}\right) & \\
& +\sum_{j<k}\left(\mu_{j k} \otimes\left(\alpha^{j} \wedge \alpha^{k}\right)+\nu_{j k} \otimes\left(\beta^{j} \wedge \beta^{k}\right)\right),
\end{aligned}
$$

where $\theta_{j k}, \mu_{j k}, \nu_{j k}, \rho_{j}, \sigma_{j}(1 \leq j, k \leq m)$, are left-invariant 1 -forms on $G_{m}(\lambda)$ satisfying $\mu_{j k}=-\mu_{k j}, \nu_{j k}=-\nu_{k j}$ and the equations (4.8), (4.9), (4.10), (4.11) and (4.12) with $\varepsilon=0$.

In particular, putting $\theta_{j k}=\mu_{j k}=\nu_{j k}=\rho_{j}=\sigma_{j}=0$ in the above theorem, we obtain that $S=0$ is a homogeneous Lorentzian structure on $\left(G_{m}(\lambda), g_{0}\right)$ and hence we have

Corollary 4.2. For each $\lambda=\left(\lambda_{1}, \ldots, \lambda_{m}\right),\left(G_{m}(\lambda), g_{0}\right)$ is a Lorentzian symmetric space.

If $\varepsilon \neq 0$, equations (4.3) and (4.4) are equivalent respectively to

$$
\begin{array}{cc}
\rho_{j}=-\beta^{j} / 2, & \sigma_{j}=\alpha^{j} / 2, \\
\theta_{j k}=\theta_{k j}, & \mu_{j k}=\nu_{j k} .
\end{array}
$$

By using (4.2), (4.5), (4.6), (4.7), (4.8), (4.9), (4.13) and (4.14), we obtain

Theorem 4.3. All the homogeneous Lorentzian structures on the oscillator group $G_{m}(\lambda)$ with the left-invariant Lorentzian metric $g_{\varepsilon}$ defined by $(3.1), \varepsilon \neq 0$, 
are given by

$$
\begin{aligned}
S=\frac{\varepsilon}{2} \sum_{i=1}^{m}\left(\beta^{i} \otimes(\eta\right. & \left.\left.\wedge \alpha^{i}\right)-\alpha^{i} \otimes\left(\eta \wedge \beta^{i}\right)\right)+\frac{1}{2} \sum_{i=1}^{m}\left(\alpha^{i} \otimes\left(\beta^{i} \wedge \xi\right)-\beta^{i} \otimes\left(\alpha^{i} \wedge \xi\right)\right) \\
& +\sum_{j, k=1}^{m} \theta_{j k} \otimes\left(\alpha^{j} \wedge \beta^{k}\right)+\sum_{j<k} \mu_{j k} \otimes\left(\alpha^{j} \wedge \alpha^{k}+\beta^{j} \wedge \beta^{k}\right)
\end{aligned}
$$

where $\theta_{j k}$ and $\mu_{j k}(1 \leq j, k \leq m)$ are left-invariant 1 -forms on $G_{m}(\lambda)$ satisfying $\theta_{j k}=\theta_{k j}, \mu_{j k}=-\mu_{k j}$, and, being $\widetilde{\nabla}=\nabla-S$,

$$
\begin{aligned}
& \widetilde{\nabla} \theta_{j k}=\sum_{i}\left(\theta_{i k} \wedge \mu_{j i}+\mu_{i k} \wedge \theta_{j i}\right)+\left(\lambda_{j}-\lambda_{k}\right) \xi \otimes \mu_{j k} \\
& \widetilde{\nabla} \mu_{j k}=\sum_{i}\left(\mu_{i k} \wedge \mu_{j i}+\theta_{j i} \wedge \theta_{k i}\right)+\left(\lambda_{k}-\lambda_{j}\right) \xi \otimes \theta_{j k}
\end{aligned}
$$

Remark 4.4. If a connected pseudo-Riemannian manifold admits a nonzero homogeneous pseudo-Riemannian structure of type $\mathcal{S}_{1}$ then it must have constant curvature (see [3] and [9]). Thus $\left(G_{m}(\lambda), g_{\varepsilon}\right), \varepsilon \neq 0$, does not admit any homogeneous Lorentzian structure of type $\mathcal{S}_{1}$. The Lorentzian symmetric space $\left(G_{m}(\lambda), g_{0}\right)$ does not admit nonzero homogeneous Lorentzian structures of type $\mathcal{S}_{1}$ either, since $\left(G_{m}(\lambda), g_{0}\right)$ has not constant curvature.

An orthonormal basis of $\left(\mathfrak{g}_{m}(\lambda),\langle,\rangle_{\varepsilon}\right)$ is $\left\{(2-2 \varepsilon)^{-1 / 2}(P-Q), X_{1}, \ldots, X_{m}\right.$, $\left.Y_{1}, \ldots, Y_{m},(2+2 \varepsilon)^{-1 / 2}(P+Q)\right\}$. Suppose $\varepsilon \neq 0$. By $(2.2)$,

$$
\begin{array}{r}
c_{12}(S)(Z)=-\frac{1}{2-2 \varepsilon} S_{P-Q, P-Q, Z}+\sum_{j=1}^{m}\left(S_{X_{j} X_{j} Z}+S_{Y_{j} Y_{j} Z}\right)+\frac{1}{2+2 \varepsilon} S_{P+Q, P+Q, Z} \\
=\sum_{j, k=1}^{m}\left(\mu_{j k}\left(X_{j}\right) \alpha^{k}(Z)+\theta_{j k}\left(X_{j}\right) \beta^{k}(Z)-\theta_{j k}\left(Y_{j}\right) \alpha^{k}(Z)+\mu_{j k}\left(Y_{j}\right) \beta^{k}(Z)\right) .
\end{array}
$$

for all $Z \in \mathfrak{g}_{m}(\lambda)$. We have (compare with [4, Prop. 2.1] for Heisenberg groups).

Proposition 4.5. A homogeneous Lorentzian structure on $\left(G_{m}(\lambda), g_{\varepsilon}\right), \varepsilon \neq 0$, is of type $\mathcal{S}_{2} \oplus \mathcal{S}_{3}$ if and only if

$$
\sum_{j=1}^{m}\left(\mu_{j k}\left(X_{j}\right)-\theta_{j k}\left(Y_{j}\right)\right)=\sum_{j=1}^{m}\left(\theta_{j k}\left(X_{j}\right)+\mu_{j k}\left(Y_{j}\right)\right)=0, \quad 1 \leq k \leq m .
$$

\section{Reductive decompositions and groups of iso- metries of the 4-dimensional oscillator group}

For each $\lambda \in \mathbb{R}$, we can consider the Lie algebra $\mathfrak{g}_{1}(\lambda)$ with generators $P, X, Y, Q$, and structure equations $[X, Y]=P,[Q, X]=\lambda Y,[Q, Y]=-\lambda X$. 
In particular, $\mathfrak{g}_{1}(0)$ is the direct product of the 3-dimensional Heisenberg algebra and $\mathbb{R}$. If $\lambda \neq 0$, then $\mathfrak{g}_{1}(\lambda)$ is isomorphic to $\mathfrak{g}=\mathfrak{g}_{1}(1)$ and the corresponding Lie group $G_{1}(\lambda)$ is isomorphic to $G=G_{1}(1)$.

Let $\{\eta, \alpha, \beta, \xi\}$ be the basis dual to $\{P, X, Y, Q\}$. We have

Theorem 5.1. All the homogeneous Lorentzian structures on the 4-dimensional oscillator group $\left(G, g_{\varepsilon}\right),-1<\varepsilon<1, \varepsilon \neq 0$, are given by

$S=\frac{\varepsilon}{2} \beta \otimes(\eta \wedge \alpha)-\frac{\varepsilon}{2} \alpha \otimes(\eta \wedge \beta)-\frac{1}{2} \beta \otimes(\alpha \wedge \xi)+\frac{1}{2} \alpha \otimes(\beta \wedge \xi)+\theta \otimes(\alpha \wedge \beta)$,

where $\theta=a \eta+b \xi, a, b \in \mathbb{R}$.

Proof. By Theorem 4.3, all the Lorentzian homogeneous structures on $G$ are given by (5.1), where $\theta$ is a 1 -form on $G$ satisfying $\widetilde{\nabla} \theta=0$. In this case,

$$
\widetilde{\nabla}_{Z} \theta=\theta(X)\left(-\theta(Z)+\frac{1}{2} \xi(Z)-\frac{\varepsilon}{2} \eta(Z)\right) \beta+\theta(Y)\left(\theta(Z)+\frac{\varepsilon}{2} \eta(Z)-\frac{1}{2} \xi(Z)\right) \alpha .
$$

Replacing $Z$ by $X$ and $Y$, the condition $\widetilde{\nabla} \theta=0$ implies $\theta(X)=0$ and $\theta(Y)=0$, respectively. Then $\theta=a \eta+b \xi, a, b \in \mathbb{R}$. Conversely, if $\theta=a \eta+b \xi$ then $\widetilde{\nabla} \theta=0$, which proves the theorem.

The nonvanishing components of the $(1,2)$ tensor field corresponding to the tensor field $S$ in (5.1) are given by

$$
\begin{array}{llll}
S_{X} P=-\frac{\varepsilon}{2} Y, & S_{P} X=a Y, & S_{P} Y=-a X, & S_{X} Q=-\frac{1}{2} Y, \\
S_{Y} P=\frac{\varepsilon}{2} X, & S_{Y} X=-\frac{1}{2} P, & S_{X} Y=\frac{1}{2} P, & S_{Y} Q=\frac{1}{2} X, \\
S_{Q} X=b Y, & S_{Q} Y=-b X . &
\end{array}
$$

From Proposition 4.5, the definitions of the classes in $\S 2$, and the characterization of connected simply connected pseudo-Riemannian naturally reductive spaces in [3], we deduce

Proposition 5.2. For every $a, b \in \mathbb{R}$, the homogeneous Lorentzian structure $S=S_{(a, b)}$ on the 4-dimensional oscillator group $\left(G, g_{\varepsilon}\right), \varepsilon \neq 0$, given by (5.1) is of type $\mathcal{S}_{2} \oplus \mathcal{S}_{3}$. Moreover, $S_{(a, b)}$ is of type $\mathcal{S}_{2}$ if and only if $a=-\varepsilon$ and $b=-1$, and of type $\mathcal{S}_{3}$ if and only if $a=\varepsilon / 2$ and $b=1 / 2$. In particular, $\left(G, g_{\varepsilon}\right)$ is a naturally reductive Lorentzian space.

The metric $g_{\varepsilon}$ is geodesically complete (see [6]) and thus every homogeneous Lorentzian structure $S_{(a, b)}$ on $\left(G, g_{\varepsilon}\right)$ has a corresponding group of isometries $\tilde{G}_{(a, b)}$ acting transitively and effectively on $G$, and an associated reductive decomposition $\tilde{\mathfrak{g}}_{(a, b)} \equiv \tilde{\mathfrak{h}}_{(a, b)} \oplus \mathfrak{g}$, where $\tilde{\mathfrak{h}}_{(a, b)}$ is the Lie algebra (isomorphic to the holonomy algebra of the connection $\left.\widetilde{\nabla}_{(a, b)}=\nabla-S_{(a, b)}\right)$ generated by the curvature operators $\left(\tilde{R}_{(a, b)}\right)_{Z W} \in \mathfrak{s o}_{1}(\mathfrak{g}), Z, W \in \mathfrak{g}$. Here, $\mathfrak{s o}_{1}(\mathfrak{g})$ is the algebra (isomorphic to $\left.\mathfrak{s o}_{1}(4)\right)$ of skew-symmetric endomorphisms of $\left(\mathfrak{g},\langle,\rangle_{\varepsilon}\right)$. The structure of Lie algebra of $\tilde{\mathfrak{g}}_{(a, b)}$ is given by

$$
\begin{aligned}
& {\left[A, A^{\prime}\right]=A A^{\prime}-A^{\prime} A, \quad A, A^{\prime} \in \tilde{\mathfrak{h}}_{(a, b)}, \quad[A, Z]=A(Z), \quad A \in \tilde{\mathfrak{h}}_{(a, b)}, Z \in \mathfrak{g},} \\
& {[Z, W]=\left(\tilde{R}_{(a, b)}\right)_{Z W}+\left(S_{(a, b)}\right)_{Z} W-\left(S_{(a, b)}\right)_{W} Z, Z, W \in \mathfrak{g} .}
\end{aligned}
$$


With respect to the basis $\{P, X, Y, Q\}$ of $\mathfrak{g}$, the connection $\widetilde{\nabla}=\widetilde{\nabla}_{(a, b)}$ is given by

$$
\widetilde{\nabla}_{P} X=-\left(\frac{\varepsilon}{2}+a\right) Y, \widetilde{\nabla}_{P} Y=\left(\frac{\varepsilon}{2}+a\right) X, \widetilde{\nabla}_{Q} X=\left(\frac{1}{2}-b\right) Y, \widetilde{\nabla}_{Q} Y=\left(b-\frac{1}{2}\right) X,
$$

with the rest vanishing. Hence, the only nonvanishing component of the curvature tensor field is $\widetilde{R}_{X Y}=\left(\frac{\varepsilon}{2}+a\right)(\beta \otimes X-\alpha \otimes Y)$. First, we shall suppose that $a=-\varepsilon / 2$. In this case, the holonomy algebra of $\widetilde{\nabla}$ is trivial and the reductive decomposition associated to the homogeneous Lorentzian structure given by $(5.1)$ is $\tilde{\mathfrak{g}}=\{0\} \oplus \mathfrak{g}$ with structure equations $[X, Y]=P,[Q, X]=\left(b+\frac{1}{2}\right) Y$, $[Q, Y]=-\left(b+\frac{1}{2}\right) X$. Then $\tilde{\mathfrak{g}}_{\left(-\frac{\varepsilon}{2}, b\right)}=\mathfrak{g}_{1}\left(b+\frac{1}{2}\right)$ and we have

Theorem 5.3. Let $S=S_{(a, b)}$ be the homogeneous Lorentzian structure on the 4-dimensional oscillator group $G=G_{1}(1)$ defined by (5.1) and $a=-\varepsilon / 2$. For $b=-1 / 2$ the corresponding group of isometries $\tilde{G}_{\left(-\frac{\varepsilon}{2},-\frac{1}{2}\right)}$ is the direct product $G_{1}(0)$ of the 3 -dimensional Heisenberg group and $\mathbb{R}$ and for $b \neq-1 / 2$ it is the oscillator group $G_{1}\left(b+\frac{1}{2}\right)$; in particular, if $b=1 / 2$ then the group of isometries is $G$ itself. For each $b \in \mathbb{R}, \tilde{G}_{\left(-\frac{\varepsilon}{2}, b\right)}=G_{1}\left(b+\frac{1}{2}\right)$ acts simply transitively on the left on $G$, for $p, q, p^{\prime}, q^{\prime} \in \mathbb{R}, z, z^{\prime} \in \mathbb{C}$, by

$$
(p, z, q) \cdot\left(p^{\prime}, z^{\prime}, q^{\prime}\right)=\left(p+p^{\prime}+\frac{1}{2} \operatorname{Im}\left(\bar{z} e^{i q\left(b+\frac{1}{2}\right)} z^{\prime}\right), z+e^{i q\left(b+\frac{1}{2}\right)} z^{\prime}, q+q^{\prime}\right) .
$$

Now, suppose that $a \neq-\varepsilon / 2$. Then $U=\tilde{R}_{X Y}=\left(\frac{\varepsilon}{2}+a\right)(X \otimes \beta-Y \otimes \alpha)$ generates the holonomy algebra $\tilde{\mathfrak{h}}_{(a, b)}$ of $\widetilde{\nabla}_{(a, b)}$ and the reductive decomposition associated to the homogeneous Lorentzian structure $S_{(a, b)}$ is $\tilde{\mathfrak{g}}_{(a, b)} \equiv \tilde{\mathfrak{h}}_{(a, b)} \oplus \mathfrak{g}=$ $\langle\{U, P, X, Y, Q\}\rangle$ with nonvanishing brackets

$$
\begin{array}{lll}
{[U, X]=-\left(\frac{\varepsilon}{2}+a\right) Y,} & {[X, Y]=U+P,} & {[P, Y]=-\left(\frac{\varepsilon}{2}+a\right) X,} \\
{[P, X]=\left(\frac{\varepsilon}{2}+a\right) Y,} & {[U, Y]=\left(\frac{\varepsilon}{2}+a\right) X,} & {[Q, Y]=-\left(b+\frac{1}{2}\right) X,} \\
{[Q, X]=\left(b+\frac{1}{2}\right) Y .} & &
\end{array}
$$

If we put $T=U+P$ then with respect to the basis $\{T, X, Y, Q, U\}$ of $\tilde{\mathfrak{g}}_{(a, b)}$ the nonvanishing brackets are $[X, Y]=T,[Q, X]=\left(b+\frac{1}{2}\right) Y,[Q, Y]=-\left(b+\frac{1}{2}\right) X$, $[U, X]=-\left(\frac{\varepsilon}{2}+a\right) Y,[U, Y]=\left(\frac{\varepsilon}{2}+a\right) X$. If $b=-1 / 2$ then $\tilde{\mathfrak{g}}_{(a, b)}$ is the direct product of the oscillator algebra $\mathfrak{g}_{1}\left(-\left(\frac{\varepsilon}{2}+a\right)\right)$ generated by $\{T, X, Y, U\}$ and the line generated by $Q$. If $b \neq-\frac{1}{2}$ then $\tilde{\mathfrak{g}}_{(a, b)}$ is the semidirect product of the oscillator algebra $\mathfrak{g}_{1}\left(b+\frac{1}{2}\right)$ generated by $\{T, X, Y, Q\}$ and the line generated by $U$ under the homomorphism $\operatorname{ad}_{\mid \mathfrak{g}_{1}\left(b+\frac{1}{2}\right)}:\langle U\rangle \rightarrow \operatorname{Der}\left(\mathfrak{g}_{1}\left(b+\frac{1}{2}\right)\right)$. In both cases, $\tilde{\mathfrak{g}}_{(a, b)}$ may also be considered a semidirect product of the 3 -dimensional Heisenberg algebra generated by $\{T, X, Y\}$ and the plane generated by $\{Q, U\}$. The corresponding connected simply connected Lie group is the semidirect product $H_{1} \times_{\gamma} \mathbb{R}^{2}$, where $\gamma$ is the action of the additive group $\mathbb{R}^{2}$ on the 3 -dimensional Heisenberg group $H_{1}$, given by $\gamma_{(q, u)}(t, z)=\left(t, e^{i\left(\left(b+\frac{1}{2}\right) q-\left(\frac{\varepsilon}{2}+a\right) u\right)} z\right)$. If the manifold $\hat{G}_{(a, b)}=\mathbb{C} \times \mathbb{R}^{3}$ is equipped with the group operation such that the bijection $(z, p, q, u) \in \hat{G}_{(a, b)} \mapsto((p, z),(q, u-p)) \in H_{1} \times_{\gamma} \mathbb{R}^{2}$ is a group isomorphism, then 
$\hat{G}_{(a, b)}$ acts transitively and almost effectively in a natural way on $G$ as a group of isometries. The normal subgroup of elements of $\hat{G}_{(a, b)}$ which act as the identity transformation on $G$ is the discrete subspace $N=\left\{\left(0,0,0, \frac{4 \pi k}{\varepsilon+2 a}\right): k \in \mathbb{Z}\right\}$, and the quotient group $\tilde{G}_{(a, b)}=\hat{G}_{(a, b)} / N$ acts transitively and effectively on $G$. The group operation of $\tilde{G}_{(a, b)} \equiv \mathbb{C} \times \mathbb{R}^{2} \times \mathbb{S}^{1}$ is given by

$$
\begin{gathered}
\left(z, p, q, e^{i u}\right)\left(z^{\prime}, p^{\prime}, q^{\prime}, e^{i u^{\prime}}\right)=\left(z+\exp \left(i\left(\left(\frac{\varepsilon}{2}+a\right) p+\left(b+\frac{1}{2}\right) q+u\right)\right) z^{\prime},\right. \\
p+p^{\prime}+\frac{1}{2} \operatorname{Im}\left(\bar{z} \exp \left(i\left(\left(\frac{\varepsilon}{2}+a\right) p+\left(b+\frac{1}{2}\right) q+u\right)\right) z^{\prime}\right), q+q^{\prime}, \\
\exp \left(i\left(u+u^{\prime}-\frac{\varepsilon+2 a}{4} \operatorname{Im}\left(\bar{z} \exp \left(i\left(\left(\frac{\varepsilon}{2}+a\right) p+\left(b+\frac{1}{2}\right) q+u\right)\right) z^{\prime}\right)\right)\right),
\end{gathered}
$$

and we conclude

Theorem 5.4. Let $S=S_{(a, b)}$ be the homogeneous Lorentzian structure on the 4-dimensional oscillator group $G=G_{1}(1)$ defined by (5.1) and $a \neq-\varepsilon / 2$. The corresponding group of isometries is $\tilde{G}_{(a, b)}=\mathbb{C} \times \mathbb{R}^{2} \times \mathbb{S}^{1}$ with the operation defined by (5.2), which acts transitively and effectively on $G$ by

$$
\begin{gathered}
\left(z, p, q, e^{i u}\right) \cdot\left(p^{\prime}, z^{\prime}, q^{\prime}\right)=\left(p+p^{\prime}+\frac{1}{2} \operatorname{Im}\left(\bar{z} \exp \left(i\left(\left(\frac{\varepsilon}{2}+a\right) p+\left(b+\frac{1}{2}\right) q+u\right)\right) z^{\prime}\right),\right. \\
\left.z+\exp \left(i\left(\left(\frac{\varepsilon}{2}+a\right) p+\left(b+\frac{1}{2}\right) q+u\right)\right) z^{\prime}, q+q^{\prime}\right), \quad z, z^{\prime} \in \mathbb{C}, p, q, p^{\prime}, q^{\prime}, u \in \mathbb{R} .
\end{gathered}
$$

\section{References}

[1] W. Ambrose and I.M. Singer, On homogeneous Riemannian manifolds, Duke Math. J. 25 (1958) 647-669.

[2] P.M. Gadea and J.A. OubiñA, Homogeneous pseudo-Riemannian structures and homogeneous almost para-Hermitian structures, Houston J. Math. 18 (1992) 449-465.

[3] P.M. GadeA and J.A. OubiÑA, Reductive homogeneous pseudo-Riemannian manifolds, Monatsh. Math. 124 (1997) 17-34.

[4] J.C. GonzÁlez and D. ChineA, Quasi-Sasakian homogeneous structures on the generalized Heisenberg group $H(p, 1)$, Proc. Amer. Math. Soc. 105 (1989) 173184 .

[5] A.V. Levichev, Chronogeometry of an electromagnetic wave given by a biinvariant metric on the oscillator group, Siberian Math. J. 27 (1986) 237-245.

[6] A.V. Levichev, Methods of investigation of the causal structure of homogeneous Lorentz manifolds, Siberian Math. J. 31 (1990) 395-408.

[7] A. Medina, Groupes de Lie munis de métriques bi-invariantes, Tôhoku Math. J. 37 (1985) 405-421.

[8] A. Medina and P. Revoy, Les groupes oscillateurs et leurs réseaux, Manuscripta Math. 52 (1985) 81-95. 
[9] A. Montesinos Amilibia, Degenerate homogeneous pseudo-Riemannian structures of class $\mathcal{S}_{1}$ on pseudo-Riemannian manifolds, preprint.

[10] D. MÜLler and F. RICCI, On the Laplace-Beltrami operator on the oscillator group, J. Reine Angew. Math. 390 (1988) 193-207.

[11] R.F. Streater, The representations of the oscillator group, Comm. Math. Phys. 4 (1967) 217-236.

[12] F. Tricerri and L. VAnhecke, Homogeneous Structures on Riemannian Manifolds, London Math. Soc. Lect. Notes Ser. 83, Cambridge Univ. Press, Cambridge, 1983.

Anschrift der autoren:

P. M. Gadea

IMAFF

CSIC

Serrano 144

28006 Madrid

Spain

e-mail: pmgadea@iec.csic.es

\section{J. A. Oubiña}

Dep. de Xeometría e Topoloxía

Facultade de Matemáticas

Universidade de Santiago de Compostela 15706 Santiago de Compostela

Spain

e-mail: oubina@zmat.usc.es 